\title{
SUBTRACTIVE EXTENSION OF IDEALS IN SEMIRINGS
}

\author{
J. N. CHAUDHARI, B. DAVVAZ AND K. J. INGALE
}

\begin{abstract}
In this paper, we (1) obtain the $k$-closure of ideals and a characterization of subtractive extension of ideals in the semiring $\mathbb{Z}_{0}^{+}$; (2) introduce the concept of closure of an ideal $A$ of a semiring $R$ with respect to an ideal $I$ of $R$ and prove the set of all subtractive extensions of an ideal $I$ of a semiring $R$ is a complete lattice; (3) show that a subtractive extension $P$ of a $Q$-ideal $I$ in a semiring $R$ is a semiprime ideal if and only if $P / I_{(Q \cap P)}$ is a semiprime ideal in the quotient semiring $R / I_{(Q)}$
\end{abstract}

\section{INTRODUCTION}

The theory of semirings was first developed by H.S. Vandiver and he has obtained important results for these objects. Semirings constitute a fairly natural generalization of rings, with board applications in the mathematical foundation of computer science. Also, semiring theory has many applications to other branches. For example, automata theory, optimization theory, algebra of formal processes, combinatorial optimization, Baysian networks and belief propagation.

A semiring is a system consisting of a set $R$ together with two binary operations on $R$ called addition and multiplication (denoted in the usual manner) such that

(1) $R$ together with addition is a (commutative) semigroup,

(2) $R$ together with multiplication is a semigroup,

(3) there exists $0 \in R$ such that $x+0=x=0+x, x \cdot 0=0=0 \cdot x$ for each $x \in R$

(4) $a \cdot(b+c)=a \cdot b+a \cdot c$ and $(a+b) \cdot c=a \cdot c+b \cdot c$ for each $a, b, c \in R$. We suppose that all semirings in this paper are commutative.

Let $\mathbb{B}$ be any Boolean algebra. Then, $(\mathbb{B}, \cup, \cap)$ is a semiring. Let $I$ be the real interval $[0,1]$. Then, $(I, \max , \min )$ is a semiring.

2010 Mathematics Subject Classification. 16Y60, 06B23.

Key words and phrases. Semiring, subtractive ideal, $Q$-ideal, quotient semiring, subtractive extension of an ideal, semiprime ideal. 
The concept of ideal, finitely generated ideal, principal ideal, semiprime ideal in a commutative semiring with identity 1 can be defined as for commutative rings with identity 1 . Denote the sets of all non-negative, and positive integers respectively by $\mathbb{Z}_{0}^{+}$, and $\mathbb{N}$. $\mathbb{Z}_{0}^{+}$is a semiring under usual addition and multiplication of non-negative integers but it is not a ring. For $a_{1}, a_{2}, \ldots, a_{n} \in \mathbb{Z}_{0}^{+}$, we denote (i) $\left\langle a_{1}, a_{2}, \ldots, a_{n}\right\rangle=$ the ideal generated by $a_{1}, a_{2}, \ldots, a_{n}$ in the semiring $\mathbb{Z}_{0}^{+}$; (ii) $\left(a_{1}, a_{2}, \ldots, a_{n}\right)=$ g.c.d. of $a_{1}, a_{2}, \ldots, a_{n}$. An ideal $I$ of a semiring $R$ is called a subtractive ideal (= $k$-ideal) if $a, a+b \in I, b \in R$, then $b \in I$. An ideal $I$ of a semiring $R$ is called a $Q$-ideal (as introduced by P. J. Allen [2]) if there exists a subset $Q$ of $R$ such that

(1) $R=\cup\{q+I: q \in Q\}$.

(2) if $q_{1}, q_{2} \in Q$, then $\left(q_{1}+I\right) \cap\left(q_{2}+I\right) \neq \emptyset \Leftrightarrow q_{1}=q_{2}$.

Let $I$ be a $Q$-ideal of a commutative semiring $R$ with identity element. Then, $R / I_{(Q)}=\{q+I: q \in Q\}$ forms a semiring under the binary operations " $\oplus$ " and "๑" defined as follows:

$$
\left(q_{1}+I\right) \oplus\left(q_{2}+I\right)=q^{\prime}+I
$$

where $q^{\prime} \in Q$ is a unique element such that $q_{1}+q_{2}+I \subseteq q^{\prime}+I$.

$$
\left(q_{1}+I\right) \odot\left(q_{2}+I\right)=q^{\prime \prime}+I
$$

where $q^{\prime \prime} \in Q$ is a unique element such that $q_{1} q_{2}+I \subseteq q^{\prime \prime}+I$. This semiring $R / I_{(Q)}$ is called a quotient semiring of $R$ by $I$. If $q_{0} \in Q$ is the unique element such that $q_{0}+I=I$, then $q_{0}+I$ is the zero element of $R / I_{(Q)}[7$, Proposition 8.21].

If $A$ is an ideal of a semiring $R$, then $\bar{A}=\{x \in R: x+y \in A$ for some $y \in$ $A$, is called the $k$-closure of $A[6]$.

The ideal theory in the quotient of commutative semirings was studied by Atani [1]. In [4], Chaudhari and Bonde introduced the concept of the subtractive extension of an ideal in semirings. In Section 2, we obtain $k$ closure of ideals and a characterization of subtractive extension of ideals in the semiring $\left(\mathbb{Z}_{0}^{+},+, \cdot\right)$. In Section 3, we introduce the concept of closure of an ideal $A$ of a semiring $R$ with respect to an ideal $I$ of $R$ and prove the set of all subtractive extensions of an ideal $I$ of a semiring $R$ is a complete lattice. Also we show that a subtractive extension $P$ of a $Q$-ideal $I$ in a semiring $R$ is a semiprime ideal if and only if $P / I_{(Q \cap P)}$ is a semiprime ideal in the quotient semiring $R / I_{(Q)}$.

The following results will be used to prove our results.

Lemma 1.1 ([3]). Let $I=\left\langle a_{1}, a_{2}, \ldots, a_{n}\right\rangle \subseteq \mathbb{Z}_{0}^{+}$. If $\left(a_{1}, a_{2}, \ldots, a_{n}\right)=d$, then there exists a smallest $r \in \mathbb{Z}_{0}^{+}$such that $d k \in I$ for all $k \geq r$. 
Theorem 1.2. ([8, Theorem 4]) Every ideal of $\mathbb{Z}_{0}^{+}$is finitely generated.

Theorem 1.3. ([5, Proposition 2.19]) An ideal I of $\mathbb{Z}_{0}^{+}$is subtractive if and only if I is a principal ideal.

\section{2. $k$-Closure AND SUbTRACTIVE EXTEnsions of IDEALS IN THE SEMIRING $\mathbb{Z}_{0}^{+}$}

In this section, we obtain the $k$-closure of ideals and a characterization of the subtractive extension of ideals in the semiring $\left(\mathbb{Z}_{0}^{+},+, \cdot\right)$.

Lemma 2.1. Let $I=\left\langle b_{1}, b_{2}, \ldots, b_{m}\right\rangle$ be a non-zero ideal of $R=\left(\mathbb{Z}_{0}^{+},+, \cdot\right)$ and $d=\left(b_{1}, b_{2}, \ldots, b_{m}\right)$. Then, $\bar{I}=\langle d\rangle$.

Proof. Let $x \in \bar{I}$. Then, $x+i \in I$ for some $i \in I$. Since $d \mid b_{j}$ for all $j, I$ $=\left\langle b_{1}, b_{2}, \ldots, b_{m}\right\rangle \subseteq\langle d\rangle$. Hence, $x+i \in\langle d\rangle$ where $i \in I \subseteq\langle d\rangle$. By Theorem 1.3, $x \in\langle d\rangle$. Now, $\bar{I} \subseteq\langle d\rangle$. For the other inclusion, by Lemma 1.1, there exists $r \in \mathbb{Z}_{0}^{+}$such that $d k \in I$ for all $k \geq r$. Then, $d r+d=d(r+1), d r \in I$. Hence, $d \in \bar{I}$. So $\langle d\rangle \subseteq \bar{I}$. Therefore, $\bar{I}=\langle d\rangle$.

Let $I$ be an ideal of a semiring $R$. An ideal $A$ of $R$ with $I \subseteq A$ is said to be a subtractive extension of $I$ if $x \in I, x+y \in A, y \in R$, then $y \in A$ [4]. Clearly, every subtractive ideal $A$ of a semiring $R$ containing an ideal $I$ of $R$ is a subtractive extension of $I$. Also every ideal of a semiring $R$ is a subtractive extension of $\{0\}$.

Example 2.2. Let $I=\{0\}, A=\langle 2,3\rangle$ be ideals in the semiring $R=$ $\left(\mathbb{Z}_{0}^{+},+, \cdot\right)$. Then, $A$ is a subtractive extension of $I$ but by Theorem 1.3. it is not a subtractive ideal.

By Theorem 1.2, every ideal of $\left(\mathbb{Z}_{0}^{+},+, \cdot\right)$ is finitely generated. Now, the following theorem gives a characterization of subtractive extensions of nonzero ideals in the semiring $\left(\mathbb{Z}_{0}^{+},+, \cdot\right)$ :

Theorem 2.3. Let $I=\left\langle b_{1}, b_{2}, \ldots, b_{m}\right\rangle$ be a non-zero ideal of $R=\left(\mathbb{Z}_{0}^{+},+, \cdot\right)$ and $d=\left(b_{1}, b_{2}, \ldots, b_{m}\right)$. Then, an ideal $A$ of $R$ is a subtractive extension of $I$ if and only if $A=\langle a\rangle$ where $a \mid d$.

Proof. Let $A$ be a subtractive extension of $I$. Suppose that $A$ is not a principal ideal. Then, by Theorem $1.2, A=\left\langle a_{1}, a_{2}, \ldots, a_{n}\right\rangle$ where $1<a_{1}<$ $a_{2}<\cdots<a_{n}, a_{i} \nmid a_{j}$ for all $i<j, j=2,3, \ldots, n, n \geq 2$. Let $d^{\prime}=$ $\left(a_{1}, a_{2}, \ldots, a_{n}\right)$. By Lemma 1.1, there exist $r_{1}, r_{2} \in \mathbb{N}$ such that $d k \in I$ and $d^{\prime} s \in A$ for all $k \geq r_{1}, s \geq r_{2}$. Hence, $d k \in I$ and $d^{\prime} k \in A$ for all $k \geq r$ where $r=\max \left\{r_{1}, r_{2}\right\} \ldots$ (1). Since $d, d^{\prime}>0, d^{\prime} r, d r+1 \geq r$. So by (1), $d d^{\prime} r \in I$ and $d d^{\prime} r+d^{\prime}=d^{\prime}(d r+1) \in A$. Since $A$ is a subtractive extension of $I, d^{\prime} \in A$. Hence, $d^{\prime}=a_{1}$. So $a_{1} \mid a_{2}$ a contradiction. Now, $A=\langle a\rangle$ for 
some $a \in \mathbb{Z}_{0}^{+}$. Since $I \subseteq A, a \mid d$. Conversely, suppose that $A=\langle a\rangle$ where $a \mid d$. Clearly, $I \subseteq A$. By Theorem 1.3, $A$ is a subtractive ideal of $R$ and hence $A$ is a subtractive extension of $I$.

The following example shows that the sum (union) of two subtractive extensions of $I$ need not be subtractive extension of $I$.

Example 2.4. Let $I=\langle 12,18\rangle, A=\langle 2\rangle$ and $B=\langle 3\rangle$ be ideals in the semiring $\mathbb{Z}_{0}^{+}$. By Theorem 2.3, $A, B$ are subtractive extensions of $I$, but $A$ $+B=\{0,2,3,4,5,6, \ldots\}=\langle 2,3\rangle$, is not a subtractive extension of $I$. An inspection will show that $A \cup B$ is not an ideal of $\mathbb{Z}_{0}^{+}$and hence $A \cup B$ is not a subtractive extension of $I$.

Denote $\mathbb{Z}_{0}^{+} \times \mathbb{Z}_{0}^{+}=\left(\mathbb{Z}_{0}^{+},+, \cdot\right) \times\left(\mathbb{Z}_{0}^{+},+, \cdot\right)$, the semiring with pointwise addition and pointwise multiplication. By [4, Lemma 2.1], $I$ is an ideal in $\mathbb{Z}_{0}^{+} \times \mathbb{Z}_{0}^{+}$if and only if $I=I_{1} \times I_{2}$ where $I_{1}, I_{2}$ are ideals in $\mathbb{Z}_{0}^{+}$.

Theorem 2.5. Let $I=I_{1} \times I_{2} \subseteq A=A_{1} \times A_{2}$ be ideals in the semiring $\mathbb{Z}_{0}^{+} \times \mathbb{Z}_{0}^{+}$. Then, $A$ is a subtractive extension of $I$ if and only if $A_{i}$ is a subtractive extension of $I_{i}$ in $\mathbb{Z}_{0}^{+}$where $i=1$, 2 .

Proof. Let $A$ be a subtractive extension of $I$ and $x \in I_{1}, x+y \in A_{1}, y \in \mathbb{Z}_{0}^{+}$. Then, $(x, 0) \in I$ and $(x, 0)+(y, 0)=(x+y, 0) \in A_{1} \times A_{2}=A$. Since $A$ is a subtractive extension of $I,(y, 0) \in A$ and hence $y \in A_{1}$. Now, $A_{1}$ is a subtractive extension of $I_{1}$. Similarly, $A_{2}$ is a subtractive extension of $I_{2}$. Conversely, suppose that $A_{i}$ is a subtractive extension of $I_{i}(\mathrm{i}=1,2)$. Clearly, $A$ is a subtractive extension of $I$.

Lemma 2.6. Let $I=I_{1} \times I_{2}$ be an ideal of the semiring $\mathbb{Z}_{0}^{+} \times \mathbb{Z}_{0}^{+}$where $I_{i}$ $\left(i=1\right.$, 2) are ideals in the semiring $\mathbb{Z}_{0}^{+}$. Then, $\bar{I}=\overline{I_{1}} \times \overline{I_{2}}$.

Theorem 2.7. Let $I=\left\langle a_{1}, a_{2}, \ldots, a_{n}\right\rangle \times\left\langle b_{1}, b_{2}, \ldots, b_{m}\right\rangle$ be an ideal of the semiring $\mathbb{Z}_{0}^{+} \times \mathbb{Z}_{0}^{+}$where $a_{i} \neq 0, b_{j} \neq 0$ for some $i$, $j$. If $d=\left(a_{1}, a_{2}, \ldots, a_{n}\right)$ and $d^{\prime}=\left(b_{1}, b_{2}, \ldots, b_{m}\right)$, then $\bar{I}=\langle d\rangle \times\left\langle d^{\prime}\right\rangle$.

Proof. Follows from Lemma 2.1 and Lemma 2.6.

\section{Subtractive EXtension of IDEAls In SEMIRINGS}

In this section, we introduce the concept of closure of an ideal $A$ of a semiring $R$ with respect to an ideal $I$ of $R$ and prove the set of all subtractive extensions of an ideal $I$ of a semiring $R$ is a complete lattice. Also we show that a subtractive extension $P$ of a $Q$-ideal $I$ in a semiring $R$ is a semiprime ideal if and only if $P / I_{(Q \cap P)}$ is a semiprime ideal in the quotient semiring $R / I_{(Q)}$. 
Let $I \subseteq A$ be ideals of a semiring $R$. Then, we denote $\overline{A_{I}}=\{x \in R$ : $x+i \in A$ for some $i \in I\}$, and will be called the closure of $A$ with respect to $I$.

We can easily show that (i) $I \subseteq \bar{I} \subseteq \overline{A_{I}} \subseteq \bar{A}$; (ii) $\overline{A_{A}}=\bar{A}$ where $I \subseteq A$ are ideals of $R$.

Example 3.1. Let $I=\{0\} \times\langle 4,6\rangle, A=\langle 2,3\rangle \times\langle 4,6\rangle$ be ideals in the semiring $R=\mathbb{Z}_{0}^{+} \times \mathbb{Z}_{0}^{+}$. Clearly, $\overline{\{0\}}=\{0\}$. By Lemma $2.1, \overline{\langle 4,6\rangle}=\langle 2\rangle$ and $\overline{\langle 2,3\rangle}=\langle 1\rangle=\mathbb{Z}_{0}^{+}$. Hence, by Lemma $2.6, \bar{I}=\{0\} \times\langle 2\rangle$ and $\bar{A}=\mathbb{Z}_{0}^{+} \times\langle 2\rangle$. An inspection will show that $\overline{A_{I}}=\langle 2,3\rangle \times\langle 2\rangle$. Now, $I \subsetneq \bar{I} \subsetneq \overline{A_{I}} \subsetneq \bar{A}$.

Theorem 3.2. Let $I \subseteq A$ be ideals of a semiring $R$. Then, $\overline{A_{I}}$ is the smallest subtractive extension of I containing A.

Proof.

(1) Let $a_{1}, a_{2} \in \overline{A_{I}}$ and $r \in R$. Then, there exist $i_{1}, i_{2} \in I$ such that $a_{1}+i_{1}, a_{2}+i_{2} \in A$. Hence, $\left(a_{1}+a_{2}\right)+\left(i_{1}+i_{2}\right)=a_{1}+i_{1}+a_{2}+i_{2} \in A$ where $i_{1}+i_{2} \in I$. So $a_{1}+a_{2} \in \overline{A_{I}}$. Similarly, $r a_{1} \in \overline{A_{I}}$. Hence, $\overline{A_{I}}$ is an ideal of $R$.

(2) Clearly, $A \subseteq \overline{A_{I}}$.

(3) Let $i \in I, a+i \in \overline{A_{I}}, a \in S$. Then, there exists $i^{\prime} \in I$ such that $a+i+i^{\prime} \in A$. Now, $i+i^{\prime} \in I$ implies $a \in \overline{A_{I}}$. Hence, $\overline{A_{I}}$ is a subtractive extension of $I$.

(4) Let $J$ be a subtractive extension of $I$ containing $A$ and let $x \in \overline{A_{I}}$. Then, there exists $i \in I$ such that $x+i \in A \subseteq J$. Since $J$ is a subtractive extension of $I, x \in J$. Hence, $\overline{A_{I}} \subseteq J$.

Corollary 3.3. Let $I \subseteq A$ be ideals of a semiring $R$. Then,

$\overline{A_{I}}=\cap\{J: J$ is a subtractive extension of $I$ in $R$ containing $A\}$.

Now, we have the following theorem:

Theorem 3.4. Let $I, A, B$ be ideals of a semiring $R$ such that $I \subseteq A, B$. Then,

(1) $A$ is a subtractive extension of $I \Leftrightarrow \overline{A_{I}}=A$.

(2) $\overline{\left(\overline{A_{I}}\right)_{I}}=\overline{A_{I}}$.

(3) $A \subseteq B \Rightarrow \overline{A_{I}} \subseteq \overline{B_{I}}$.

(4) $\overline{(A \cap B)_{I}}=\overline{A_{I}} \cap \overline{B_{I}}$.

(5) If $A, B$ are subtractive extensions of $I$, then $A \cap B$ is a subtractive extension of $I$.

(6) If $J$ is an ideal of $R$ such that $I \subseteq J \subseteq A$, then $\overline{A_{I}} \subseteq \overline{A_{J}}$. 
Proof.

(1) Follows from Theorem 3.2.

(2) Follows from Theorem 3.2 and (1).

(3) Let $A \subseteq B$ and let $x \in \overline{A_{I}}$. Then, there exists $i \in I$ such that $x+i \in A \subseteq B$. Hence, $x \in \overline{B_{I}}$. Now, $\overline{A_{I}} \subseteq \overline{B_{I}}$.

(4) Since $A \cap B \subseteq A, \overline{(A \cap B)_{I}} \subseteq \overline{A_{I}}$. Similarly, $\overline{(A \cap B)_{I}} \subseteq \overline{B_{I}}$. Now, $\overline{(A \cap B)_{I}} \subseteq \overline{A_{I}} \cap \overline{B_{I}}$. For the other inclusion, let $x \in \overline{A_{I}} \cap \overline{B_{I}}$. Then, there exist $i_{1}, i_{2} \in I$ such that $x+i_{1} \in A$ and $x+i_{2} \subseteq B$. Since $i_{2} \in I \subseteq A$ and $A$ is an ideal of $R, x+i_{1}+i_{2} \in A$. Similarly, $x+i_{1}+i_{2} \in B$. Now, $x+i_{1}+i_{2} \in A \cap B$. So $x \in \overline{(A \cap B)_{I}}$. Hence, $\overline{A_{I}} \cap \overline{B_{I}} \subseteq \overline{(A \cap B)_{I}}$.

(5) By (4), $\overline{(A \cap B)_{I}}=\overline{A_{I}} \cap \overline{B_{I}}=A \cap B$, since $A, B$ are subtractive extensions of $I$. So by (1), $A \cap B$ is a subtractive extension of $I$.

(6) Suppose that $J$ is an ideal of $R$ such that $I \subseteq J \subseteq A$. Let $x \in \overline{A_{I}}$. Then, $x+i \in A$ for some $i \in I \subseteq J$. Hence, $x \in \overline{A_{J}}$. Now, we have $\overline{A_{I}} \subseteq \overline{A_{J}}$.

Now, by Theorem 3.4, we have

(i) $A$ is a subtractive ideal of $R$ if and only if $\bar{A}=A$.

(ii) $\overline{\bar{A}}=\bar{A}$.

(iii) $A \subseteq B$ implies $\bar{A} \subseteq \bar{B}$.

Corollary 3.5. Let $A, B$ be ideals of a semiring $R$. Then, $\overline{A \cap B} \subseteq \bar{A} \cap \bar{B}$.

Proof. By using Theorem 3.4 (3) and Theorem 3.4 (6), we have,

$$
\begin{aligned}
\overline{A \cap B} & =\overline{(A \cap B)_{(A \cap B)}} \\
& \subseteq \overline{A_{(A \cap B)}} \\
& \subseteq \overline{A_{A}} \\
& =\bar{A} .
\end{aligned}
$$

Similarly, $\overline{A \cap B} \subseteq \bar{B}$. Now, $\overline{A \cap B} \subseteq \bar{A} \cap \bar{B}$.

The following example shows that the equality in Corollary 3.5 may not

\begin{tabular}{|c|c|c|c|c|}
\hline+ & $0 a b$ & & & $0 a b$ \\
\hline 0 & $0 a b$ & and & 0 & $\begin{array}{lll}0 & 0 & 0\end{array}$ \\
\hline$a$ & $a a b$ & dI & $a$ & $\begin{array}{lll}0 & 0 & 0\end{array}$ \\
\hline$b$ & $b b b$ & & $b$ & $00 b$ \\
\hline
\end{tabular}
hold.

Example 3.6. Let $R=\{0, a, b\}$. Then, $R$ forms a semiring under the following addition and multiplication: 
Here $A=\{0, a\}, B=\{0, b\}$ are ideals of $R$. Clearly, $\overline{A \cap B}=\{0\}$. An inspection will show that $\bar{A}=A$ and $\bar{B}=R$. Hence, $\bar{A} \cap \bar{B}=A$. Now, $\overline{A \cap B} \subsetneq \bar{A} \cap \bar{B}$.

Theorem 3.7. Let $I$ be an ideal of a semiring $R$. If $\mathcal{A}(I)$ denotes the set of all subtractive extensions of $I$ in $R$, then $\mathcal{A}(I)$ is a complete lattice.

Proof. Clearly, $(\mathcal{A}(I), \subseteq)$ is a partially ordered set. Let $A, B \in \mathcal{A}(I)$. Then, by Theorem $3.4(5), A \cap B \in \mathcal{A}(I)$ and by using Theorem $3.2, \overline{(A+B)_{I}} \in$ $\mathcal{A}(I)$. Define $A \wedge B=A \cap B$ and $A \vee B=\overline{(A+B)_{I}}$. Let $C \in \mathcal{A}(I)$ such that $A, B \subseteq C$. Then, $A+B \subseteq C$ and hence $\overline{(A+B)_{I}} \subseteq \overline{C_{I}}=C$ since by Theorem $3.4(1)$. Now, $\overline{(A+B)_{I}}$ is a l.u.b. of $A, B$. Hence, $\mathcal{A}(I)$ is a complete lattice.

Corollary 3.8. Let $I$ be an ideal of a semiring $R$. If $\mathcal{K}(I)$ denotes the set of all subtractive ideals of $R$ containing $I$, then $\mathcal{K}(I)$ is a complete lattice.

Let $I \subseteq A$ be ideals of a semiring $R$. If $I$ is a $Q$-ideal of $R$, then $\widetilde{A}=$ $\left\{x \in R\right.$ : there exists $q+I \in R / I_{(Q)}$ such that $x \in q+I$ and $\left.(q+I) \cap A \neq \emptyset\right\}$, is called the closure of $A$ with respect to $I_{(Q)}$ [4].

In the next lemma we give the relation between $\overline{A_{I}}$ and $\widetilde{A}$.

Lemma 3.9. Let $I$ be a $Q$-ideal of a semiring $R$ and $A$ be an ideal of $R$ with $I \subseteq A$. Then, $\overline{A_{I}}=\widetilde{A}$.

Proof. Let $x \in \overline{A_{I}}$. Then, there exists $i_{1} \in I$ such that $x+i_{1} \in A$...(1). By [2, Lemma 7], there exists $q \in Q$ such that $x \in q+I$. Then, $x=q+i_{2}$ for some $i_{2} \in I$. So $x+i_{i}=q+i_{2}+i_{1} \in q+I$. Thus, $(q+I) \cap A \neq \emptyset$. Hence, $x \in \widetilde{A}$. Now, $\overline{A_{I}} \subseteq \widetilde{A}$. For the other inclusion, let $z \in \widetilde{A}$. Then, there exists $q+I \in R / I_{(Q)}$ such that $z \in q+I$ and $(q+I) \cap A \neq \emptyset$. So $z=q+i^{\prime}$ for some $i^{\prime} \in I$. Let $y=q+i^{\prime \prime} \in(q+I) \cap A$ where $i^{\prime \prime} \in I$. Since $i^{\prime} \in I \subseteq A$ and $A$ is an ideal of $R, z+i^{\prime \prime}=q+i^{\prime \prime}+i^{\prime} \in A$. Hence, $z \in \overline{A_{I}}$. Now, $\widetilde{A} \subseteq \overline{A_{I}}$.

By Lemma 3.9 and Theorem 3.2, we have the following:

Theorem 3.10 ([4]). Let $I$ be a $Q$-ideal of a semiring $R$ and $A$ be an ideal of $R$ with $I \subseteq A$. Then, $\widetilde{A}$ is the smallest subtractive extension of $I$ containing $A$.

Theorem 3.11. Let $I$ be a $Q$-ideal of a semiring $R$ and $P$ a subtractive extension of $I$. Then, $P$ is a semiprime ideal of $R$ if and only if $P / I_{(Q \cap P)}$ is a semiprime ideal of $R / I_{(Q)}$.

Proof. Let $P$ be a semiprime ideal of $R$. Suppose that $q+I \in R / I_{(Q)}$ and $(q+I) \odot(q+I)=q^{\prime}+I \in P / I_{(Q \cap P)}$ where $q^{\prime} \in Q \cap P$ is a unique element such 
that $q^{2}+I \subseteq q^{\prime}+I$. So $q^{2}=q^{\prime}+i$ for some $i \in I$. Now, $q^{2} \in P$ implies $q \in P$. Hence, $q+I \in P / I_{(Q \cap P)}$. Conversely, suppose that $P / I_{(Q \cap P)}$ is a semiprime ideal of $R / I_{(Q)}$. Let $a^{2} \in P$ where $a \in R$. Since $I$ is a $Q$-ideal of $R$, there exist unique $q, q^{\prime} \in Q$ such that $a \in q+I$ and $a^{2} \in(q+I) \odot(q+I)=q^{\prime}+I$, where $q^{2}+I \subseteq q^{\prime}+I$. Thus, $a^{2}=q^{\prime}+i^{\prime}$ for some $i^{\prime} \in I$. Since $P$ is a subtractive extension of $I, q^{\prime} \in P$. Hence, $(q+I) \odot(q+I)=q^{\prime}+I \in P / I_{(Q \cap P)}$. Since $P / I_{(Q \cap P)}$ is a semiprime ideal, $q+I \in P / I_{(Q \cap P)}$. Now, $a \in q+I \Rightarrow a=q+i^{\prime \prime}$ for some $i^{\prime \prime} \in I \Rightarrow a \in P$ as $q \in Q \cap P \subseteq P$.

\section{REFERENCES}

[1] S. E. Atani, The ideal theory in quotients of commutative semirings, Glas. Mat. Ser. III, 42 (62) (2007), no. 2, 301-308.

[2] P. J. Allen, A fundamental theorem of homomorphism for semirings, Proc. Amer. Math. Soc., 21 (1969), 412-416.

[3] P. J. Allen and L. Dale, Ideal theory in the semiring $\mathbb{Z}_{0}^{+}$, Publ. Math. Debrecen, 22 (3-4) (1975), 219-224.

[4] J. N. Chaudhari and D. R. Bonde, Ideal theory in quotient semirings, Thai J. Math., 12 (1) (2014), 95-101.

[5] J. N. Chaudhari and V. Gupta, Weak primary decomposition theorem for right Noetherian semirings, Indian J. Pure Appl. Math., 25 (6) (1994), 647-654.

[6] L. Dale, The $k$-closure of monic and monic free ideals in a polynomial semiring, Proc. Amer. Math. Soc., 64 (2) (1977), 219-226.

[7] J. S. Golan, Semiring and Their Applications, Kluwer Academic publisher Dordrecht, 1999.

[8] V. Gupta and J. N. Chaudhari, Some remarks on semirings, Rad. Mat., 12 (2003), $13-18$.

[9] M. K. Sen and M. R. Adhikari, On k-ideals of semirings, Internat. J. Math. Math. Sci., 15 (2) (1992), 347-350.

(Received: October 6, 2013)

(Revised: March 14, 2014)
J. N. Chaudhari and K. J. Ingale

Department of Mathematics, M. J. College Jalgaon-425002, India

jnchaudhari@rediffmail.com

B. Davvaz

Department of Mathematics

Yazd University

Yazd, Iran

davvaz@yazd.ac.ir 